\title{
Contribution à l'étude des méthodes d'analyse automatique du lait et du lactosérum secs
}

\author{
par \\ Sonia AMARIGLIO et Annie PONDAVEN \\ Laboratoire Central de l'Inspection du Lait*
}

\section{INTRODUCTION}

Depuis quelques années, les applications de l'analyse automatique se sont considérablement développées, en particulier dans les laboratoires d'analyses biologiques. Les méthodes d'examen des produits laitiers peuvent-elles bénéficier du progrès technique que représente l'automation? Quels sont les avantages et les inconvénients occasionnés par l'utilisation de ce procédé ?

L'automation apporte une solution au problème de la standardisation des contrôles. Elle assure une répétabilité améliorée des résultats, en comparaison avec les techniques manuelles où l'appréciation subjective de l'opérateur peut intervenir. Elle permet de multiplier les examens. Les exigences croissantes de l'utilisateur, du consommateur et du législateur impliquent, au niveau de l'entreprise industrielle, une surveillance accrue à chaque stade de transformation ainsi qu'une meilleure sélection de la matière première. Il s'ensuit, pour le laboratoire de contrôle, une augmentation importante du nombre d'échantillons à analyser et du nombre de paramètres à examiner sur chacun d'eux.

Les avantages présentés par l'utilisation de l'autoanalyseur découlent essentiellement de ses caractéristiques. On peut citer :

- La vitesse de fonctionnement. En un temps donné, il est examiné un plus grand nombre d'échantillons avec cet appareillage que par les méthodes manuelles.

- La polyvalence. Avec ce système, il est possible d'effectuer des déterminations très variées, ce qui augmente sa rentabilité.

- La simplicité de mise en œuvre et d'entretien. Le passage d'un type de dosage à un autre est très rapide.

\footnotetext{
* Ministère de l'Agriculture (Direction des Services Vétérinaires). Laboratoire Central d'Hygiène Alimentaire, 43, rue Dantzig - 75015 Paris. Tél. : 531-82-10.
} 
- La fiabilité et la précision des résultats. L'automaticité des opérations leur confère un haut degré de reproductibilité. Les erreurs humaines inévitables, plus particulièrement lors des analyses de routine, sont éliminées.

- L'enregistrement sur papier des courbes de mesure. Le document obtenu limite les risques d'erreur lors de l'interprétation et permet de conserver la trace probante des résultats.

***

Nous avons commencé notre étude par l'adaptation à l'autoanalyse des méthodes traditionnelles colorimétriques employées dans notre laboratoire [1].

En effet, tout dosage dont la réaction finale peut être mesurée par colorimétrie est susceptible d'être automatisé.

Dans un premier temps, nous avons étudié l'application des techniques nécessaires à l'examen de grandes séries d'échantillons de laits secs écrémés et de lactosérums secs afin de permettre la transmission des résultats dans le délai restreint exigé.

Ces déterminations sont les suivantes :

- Acidité titrable.

- Acide lactique et lactates.

- Ammoniac.

D'autres dosages peuvent être réalisés grâce à l'autoanalyseur ; citons par exemple :

- Azote total.

- Chlorures.

- Lactose.

- Nitrites et nitrates.

- Eléments minéraux.

\section{DESCRIPTION DE L'AUTOANALYSEUR}

L'ensemble analytique est constitué de plusieurs modules. Chacun effectue une opération déterminée, les réactifs circulent d'une extrémité à l'autre de la chaîne sous l'action d'une pompe à multicanaux, passant ainsi par les étapes successives de traitement jusqu'à la mesure finale.

Il s'agit d'un système à flux continu. Les phénomènes de diffusion sont éliminés et l'intégrité des échantillons respectée grâce à une 
segmentation par de l'air. L'enregistrement ininterrompu permet le contrôle du bon fonctionnement de l'appareil à chaque stade de l'analyse.

Des étalons sont périodiquement introduits dans le système analytique. Les résultats recherchés sont obtenus par comparaison permanente avec les valeurs correspondant aux étalons de concentration connue.

L'autoanalyseur I Technicon utilisé se compose des six modules suivants (fig. 1) :

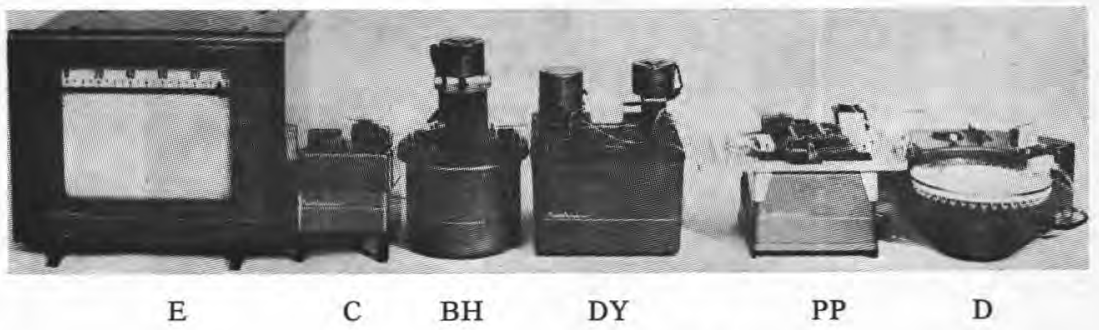

fig. 1 : Ensemble analytique Technicon

- Un distributeur (D). Les échantillons liquides sont déposés dans des godets en matière plastique. Il est donc nécessaire de diluer les produits secs.

- Une pompe proportionnante (PP). Cette pompe réalise les prises d'essai d'échantillons et de réactifs dans les proportions voulues par la méthode analytique. L'ensemble des tubes de pompage, tubes de connexion, bobines de mélange, est monté sur un plateau appelé " manifold » qui constitue le jeu d'accessoires particulier à chaque type de dosage.

- Un dialyseur (DY). La dialyse a pour objet d'éliminer les les macromolécules et les impuretés.

Dans le cas de l'analyse du lait, la dialyse remplace la défécation suivie de la filtration en éliminant : les globules gras, les protéines, les pigments de base ; le dialysat contient l'élément à doser en concentration proportionnelle à celle de l'échantillon initial. La dialyse présente un net avantage car elle supprime les interférences possibles apportées par les défécants dans les réactions colorées.

- Un bain d'huile $(\mathrm{BH})$. La régularisation de la température permet de se placer dans les conditions optimales de réaction de certains dosages.

- Un colorimètre $(C)$. La mesure finale est effectuée par colorimétrie. Le colorimètre fonctionne en flux continu après élimination 
des bulles d'air de segmentation. Les longueurs d'onde sont sélectionnées par des filtres interférentiels à bande passante étroite.

- Un enregistreur (E). Il inscrit chaque signal électrique fourni par le colorimètre. La concentration d'un constituant chimique est fonction de la hauteur des pics tracés sur le papier de l'enregistreur.

蛋

Dans la suite du texte, nous avons développé l'analyse des laits et des lactosérums écrémés en poudre.

La méthodologie proposée est également applicable aux produits liquides. Il suffit de les diluer de moitié pour se trouver dans les mêmes conditions d'essais.

Pour les produits non écrémés, la prise d'essai doit être augmentée et calculée de façon à avoir une quantité équivalente de matière non grasse.

Pour améliorer la précision des résultats, il est conseillé de procéder à un étalonnage avec un lait ou un lactosérum de composition analogue à celle des produits analysés.

\section{CONSIDERATIONS PRATIQUES GENERALES}

Le volume des prises d'essai d'échantillons et de réactifs est proportionnel au diamètre des tubes de pompage. Le débit de ces tubes $(\mathrm{ml} / \mathrm{mn})$ est indiqué sur les schémas de montage.

Les échantillons peuvent être analysés à une cadence variable : 20,30 ou 40 échantillons à l'heure avec un temps de prélèvement équivalent $(1 / 1)$ ou double $(2 / 1)$ de celui du rinçage. Dans le texte suivant, le nombre d'échantillons et le rapport prélèvement-rinçage seront indiqués de la façon suivante :

- Exemple : 20 (1/1), c'est-à-dire 20 échantillons à l'heure, temps de prélèvement identique à celui du rinçage ou 40 (2/1), c'est-à-dire 40 échantillons à l'heure, temps de prélèvement double de celui du rinçage.

L'amplitude des pics diminue quand la cadence des déterminations augmente. Un échantillon, présentant une teneur élevée du constituant analysé, peut fausser par excès le résultat de l'examen de l'échantillon suivant; la recherche d'une durée de rinçage efficace est nécessaire. 
Pour chaque dosage, il est nécessaire de faire une étude en changeant les différents paramètres afin de déterminer les conditions optimales de l'essai.

Par ailleurs, la sensibilité peut être augmentée par l'utilisation d'un expanseur d'échelle.

\section{I. - DETERMINATION DE L'ACIDE LACTIQUE ET DES LACTATES DES LAITS SECS ECREMES}

La méthode décrite ci-dessous permet de doser l'acidité lactique acquise par fermentation du lait.

La méthode employée est une transposition de la méthode Venekamp et Kruisheer [1, 3, 4].

\section{1) Principe}

L'acide lactique présent dans le lait reconstitué à 5 p. $100(\mathrm{~m} / \mathrm{v})$, réagit avec le chlorure ferrique à un $\mathrm{pH}$ voisin de 2 pour former du lactate de fer jaune dont l'intensité de coloration est mesurée à la longueur d'onde de $420 \mathrm{~nm}$ dans une cuve tubulaire à flux continu de $15 \mathrm{~mm}$. Le liquide de dilution et de contre-dialyse est une solution de chlorure de sodium isotonique.

La figure 2 représente le montage, ou manifold, particulier à ce dosage.

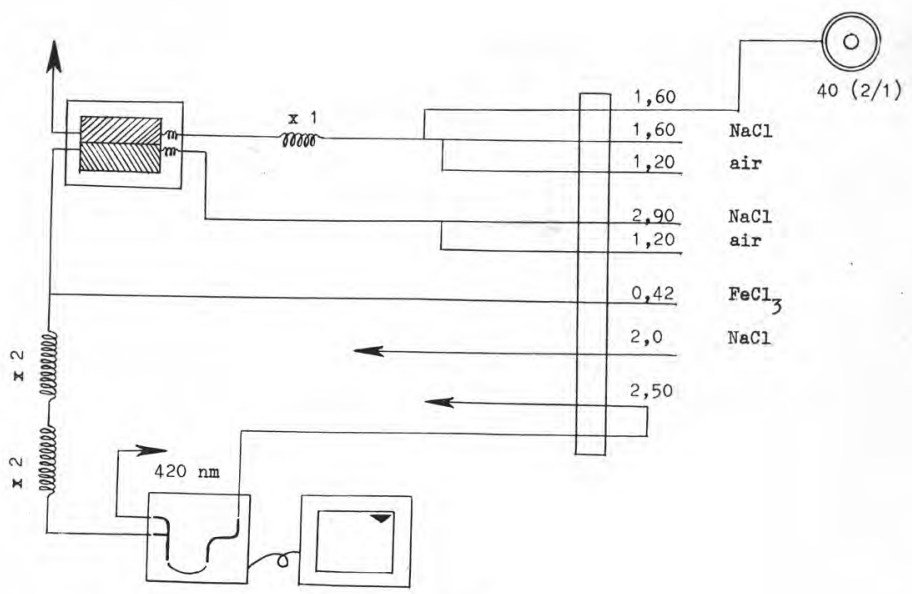

fig. 2

Diagramme des flux pour le dosage de l'acide lactique/lactactes 


\section{2) Réactifs}

2.1. Solution de chlorure de sodium :

$\mathrm{NaCl}$

$9 \mathrm{~g}$

Eau distillée q.s.p.

$1000 \mathrm{ml}$

2.2. Solution de chlorure ferrique :

Solution de perchlorure de fer à $27-29$ p 100

$10 \mathrm{ml}$

$\mathrm{HCl} \mathrm{N}$

$12 \mathrm{ml}$

Eau distillée q.s.p.

2.3. Solution de chlorure mercurique :

$\mathrm{HgCl}_{2}$

Eau distillée q.s.p.

2.4. Solutions de référence de lactate:

a) Solution mère : A.

Faire dissoudre $106,66 \mathrm{~g}$ de lactate de lithium anhydre $\left(\mathrm{CH}_{3}-\mathrm{CHOH}\right.$ COOLi) dans de l'eau distillée. Amener à $1000 \mathrm{ml}$ avec de l'eau distillée. lactique.

$1 \mathrm{ml}$ de cette solution contient l'équivalent de $100 \mathrm{mg}$ d'acide

b) Solutions diluées : B, C, D...

A partir de la solution mère A, préparer des solutions diluées en prélevant $\mathrm{X} \mathrm{ml}$ de la solution $\mathrm{A}$ et en complétant à $100 \mathrm{ml}$ à l'aide d'eau distillée.

Préparer par exemple, les solutions suivantes :

Solution B : $\quad 2,5 \mathrm{ml} \mathrm{A} / 100 \mathrm{ml}$ soit $2,5 \mathrm{mg}$ d'acide lactique par $\mathrm{ml}$.

Solution C : $\quad 5,0 \mathrm{ml} \mathrm{A} / 100 \mathrm{ml}$ soit $5,0 \mathrm{mg}$ d'acide lactique par $\mathrm{ml}$.

Solution D : $7,5 \mathrm{ml} \mathrm{A} / 100 \mathrm{ml}$ soit $7,5 \mathrm{mg}$ d'acide lactique par $\mathrm{ml}$.

Solution $\mathrm{J}$ : $22,5 \mathrm{ml} \mathrm{A} / 100 \mathrm{ml}$ soit $22,5 \mathrm{mg}$ d'acide lactique par $\mathrm{ml}$. Solution $\mathrm{K}$ : $25,0 \mathrm{ml} \mathrm{A} / 100 \mathrm{ml}$ soit $25,0 \mathrm{mg}$ d'acide lactique par $\mathrm{ml}$.

\section{3) Etalonnage}

A un lait sec, reconstitué à 5 p. $100(\mathrm{~m} / \mathrm{v})$, exempt d'acide lactique et de lactates ou de teneur aussi faible que possible, ajouter des quantités croissantes de lactate de lithium.

Préparation de la courbe d'étalonnage

- Echantillon témoin. 
2,5 $\mathrm{g}$ de lait sec (défini précédemment) sont dilués avec $49 \mathrm{ml}$ d'eau distillée. Agiter.

Ajouter $1 \mathrm{ml}$ de solution mercurique (2.3).

- Echantillon d'étalonnage.

Introduire, dans une série de 10 fioles coniques, 2,5 g du lait sec précédent.

Ajouter $48 \mathrm{ml}$ d'eau distillée, puis agiter afin d'obtenir une solution homogène.

Ajouter respectivement dans chaque fiole, $1 \mathrm{ml}$ de chacune des solutions $\mathrm{B}$ à $\mathrm{K}$. fioles.

Ajouter $1 \mathrm{ml}$ de solution mercurique (2.3) dans chacune des

Ces onze échantillons servent à l'établissement de la courbe d'étalonnage (fig. 3). Ils sont utilisés dans les mêmes conditions que les échantillons à tester pour évaluer leur teneur en acide lactique.

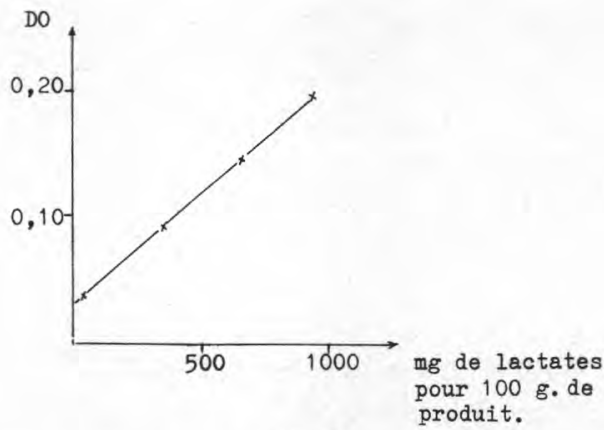

fig 3

Profil de la courbe d'étalonnage du dosage de l'acide lactique/lactates

Grâce à la présence du chlorure mercurique, agent conservateur, il est possible de les utiliser pendant 3 à 4 semaines en les maintenant le plus possible au réfrigérateur à $4^{\circ} \mathrm{C}$.

\section{4) Mise au point de la technique}

\subsection{Etude du taux de reconstitution des laits secs}

Un lait sec reconstitué à 2,5-5,0-10 p. $100(\mathrm{~m} / \mathrm{v})$ avec de l'eau distillée est enrichi en lactate de lithium.

Après dosage de l'acide lactique par l'autoanalyseur, nous constatons que la courbe (fig. 4) correspondant à la reconstitution à 5 p. $100(\mathrm{~m} / \mathrm{v})$ est linéaire et possède une pente relativement élevée. 


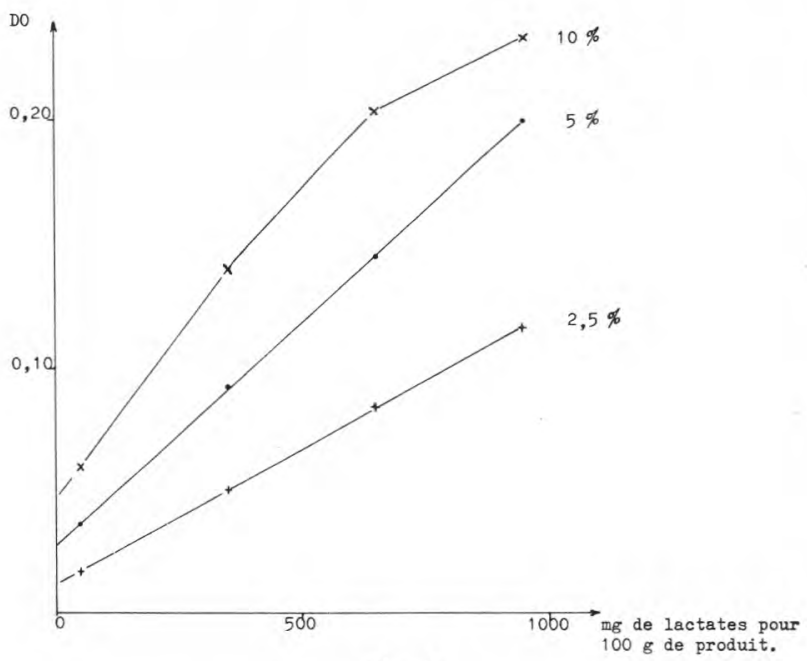

fig. 4

Recherche du taux optimal de reconstitution du lait

\subsection{Influence du réactif}

\section{- Concentration de la solution de perchlorure de fer.}

Dans la méthode préconisée par Venekamp et Kruisheer, la concentration de la solution de perchlorure de fer est de 0,5 p. 100 en $\mathrm{Cl}_{3} \mathrm{Fe}$ et le lait est reconstitué à $10 \mathrm{p} .100$.

Lors de la transposition de la méthode, la concentration de cette solution a été diminuée de moitié pour se placer dans le même rapport, le taux de reconstitution retenu en définitive pour le lait étant de 5 p. $100(\mathrm{~m} / \mathrm{v})$.

- Sensibilité de la réaction en fontion du pH de la solution de perchlorure de fer.

Ling, utilisant une méthode analogue, préconise un $\mathrm{pH}$ réactionnel de 2,0. Des essais ont été effectués en utilisant des concentrations croissante d'acide chlorhydrique normal afin d'observer l'influence du $\mathrm{pH}$ sur la sensibilité de la réaction.

L'emploi de cet acide a pour but :

- d'abaisser le $\mathrm{pH}$ de la solution de perchlorure de fer, dialysée,

- d'éviter l'encrassement lors du mélange $\mathrm{Cl}_{3} \mathrm{Fe}$ - solution réaction.

- de déterminer les conditions de sensibilité maximale de la

Dans les essais suivants, la variante est la concentration en acide chlorhydrique, tous les autres facteurs sont constants : 
Solution de perchlorure de fer à $26-29$ p. 100

$\mathrm{HCl} \mathrm{N}$

$10 \mathrm{ml}$

Eau distillée q.s.p.

L'influence de la quantité d'acide sur le pH de la solution finale est montrée dans le tableau suivant :

\begin{tabular}{r|c}
\hline $\mathrm{HCl} \mathrm{N}$ & $\mathrm{pH}$ de la solution \\
\hline & \\
$8 \mathrm{ml}$ & 2,1 \\
$12 \mathrm{ml}$ & 2,0 \\
$16 \mathrm{ml}$ & 1,9 \\
$24 \mathrm{ml}$ & 1,7 \\
$32 \mathrm{ml}$ & 1,6 \\
$40 \mathrm{ml}$ & 1,5 \\
\hline
\end{tabular}

Les résultats obtenus sont portés sur la figure 5 .

Nous constatons que les tracés correspondant aux solutions à 8 , 12 et $16 \mathrm{ml}$ d'acide chlorhydrique ont une sensibilité presque identique.

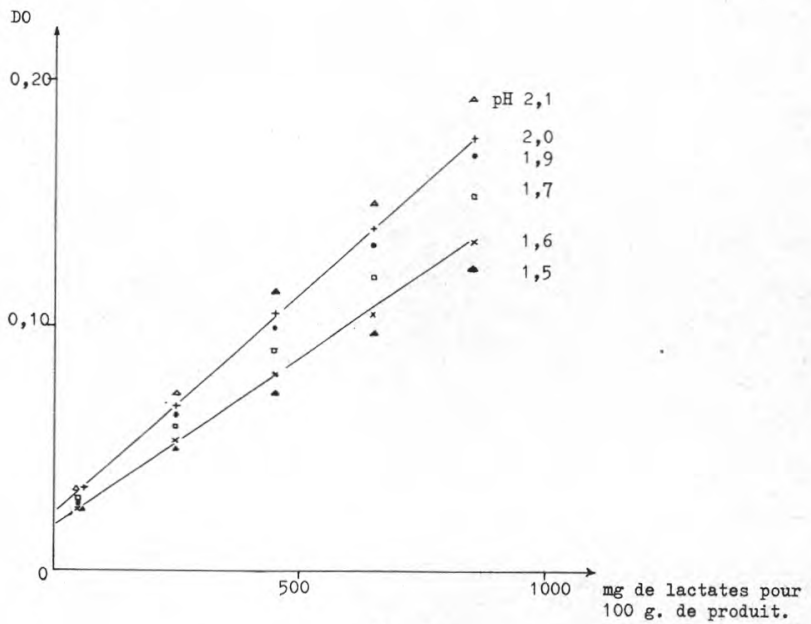

fig. 5

Influence du $\mathrm{pH}$ du réactif sur la réaction colorée

\subsection{Cadence analytique}

Des essais effectués à $50(2 / 1), 40(2 / 1)$ et $30(2 / 1)$ échantillons/h, nous retenons les cadences de 30 et 40 échantillons pour lesquelles la répétabilité des résultats est satisfaisante. 
Au rythme de 40 échantillons/h, les résultats sont facilement interprétables (fig. 6) et à cette cadence les déterminations de l'acidité titrable et de la teneur en acide lactique et lactates peuvent se pratiquer simultanément (avec cependant un appareillage approprié).
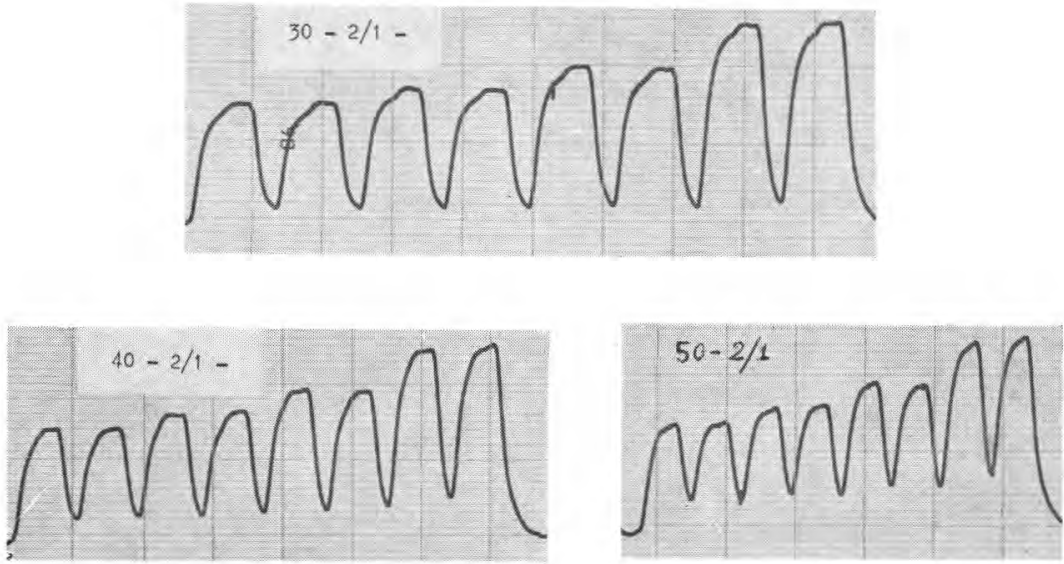

fig. 6

Courbes d'enregistrement des résultats de l'analyse de 4 laits examinés (en double) aux cadences de 30 - 40 - 50 échantillons par heure

\subsection{Fiabilité de l'appareil}

L'analyse effectuée à une cadence de 40 échantillons/h, dans les conditions définies ci-dessus, donne des résultats très satisfaisants.

En effet, pour un même échantillon présenté à 6 reprises successives, aucune variation d'amplitude n'est enregistrée (fig. 7).

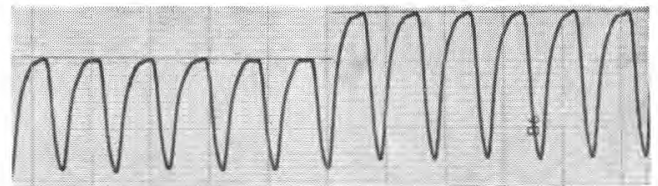

fig. 7

Fiabilité de l'appareil 


\section{5) Comparaison des méthodes}

Sur 92 échantillons analysés par la méthode Venekamp et Kruisheer, et par la méthode transposée à l'autoanalyse, les écarts relevés sont les suivants :

\begin{tabular}{c|c}
\hline Nombre d'échantillons & Ecart en mg de lactates pour $100 \mathrm{~g}$ \\
\cline { 2 - 2 } 21 & 0 \\
24 & 10 \\
15 & 20 \\
8 & 30 \\
4 & 40 \\
9 & 50 \\
1 & 60 \\
4 & 70 \\
2 & 80 \\
2 & 90 \\
2 & 100 \\
\hline
\end{tabular}

La moyenne des écarts est égale à $24,67 \mathrm{mg}$ de lactates pour $100 \mathrm{~g}$ de produit. Cet écart peut être imputé au fait que les deux méthodes analytiques présentent des différences dans leur protocole opératoire.

Lors de l'interprétation des résultats et de l'application de l'équation :

$$
\mathrm{An}+\mathrm{Al}=\mathrm{At}[1]\left\{\begin{array}{l}
\mathrm{An}: \text { Acidité naturelle } \\
\mathrm{Al}: \text { Acidité lactique } \\
\mathrm{At}: \text { Acidité titrable }
\end{array}\right.
$$

l'écart moyen de 24,67 mg peut être considéré comme négligeable, tout au moins dans l'optique d'un contrôle en grande série.

La figure 8 montre la différence obtenue entre les deux méthodes, soit :

- 64,3 p. $100 \leqslant$ à $25 \mathrm{mg} \quad$ pour $100 \mathrm{~g}$,

- 13,5 p. 100 entre $25 \mathrm{mg}$ et $50 \mathrm{mg}$ pour $100 \mathrm{~g}$,

- 15,0 p. 100 entre $50 \mathrm{mg}$ et $75 \mathrm{mg}$ pour $100 \mathrm{~g}$,

- 7,2 p. 100 entre $75 \mathrm{mg}$ et $100 \mathrm{mg}$ pour $100 \mathrm{~g}$.

Il ne semble pas qu'il y ait de relation entre la teneur en acide lactique/lactates et l'écart constaté entre les deux méthodes analytiques.

\section{6) Modalités retenues}

6.1. Taux de reconstitution du lait sec : 5 p. 100 .

6.2. Réactif : 0,25 p. 100 en $\mathrm{FeCl}_{3}$, à pH 2,0.

6.3. Cadence : $40(2 / 1)$. 


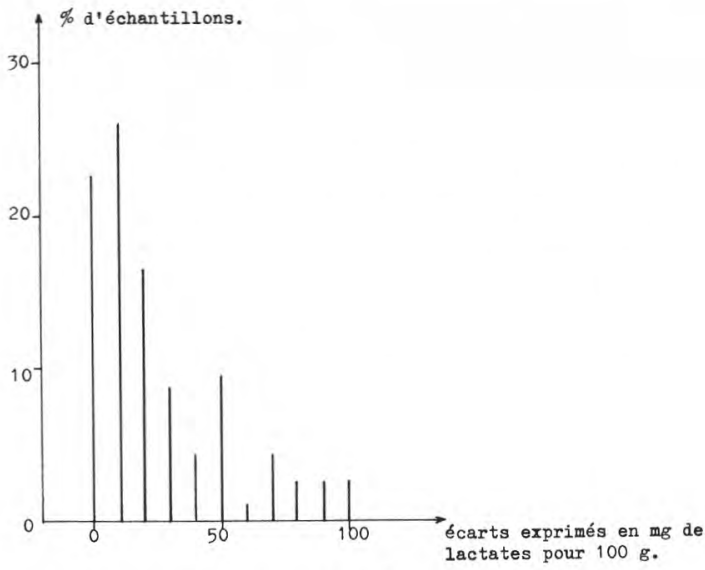

fig. 8

Répartition des écarts entre les résultats des deux méthodes analytiques

Observation : Après chaque série de déterminations, il est nécessaire de rincer le circuit avec une solution d'acide chlorhydrique à 20 p. 100 afin de dissoudre le dépôt ferrique au niveau du mélange dialysat-solution ferrique.

\section{II. - DETERMINATION DE L'ACIDITE TITRABLE DES LAITS SECS ECREMES}

La technique employée est adaptée de la méthodologie Technicon [2] pour le dosage des carbonates, bicarbonates et anhydride carbonique dissous, présents dans le sérum et le plasma. La méthode de référence [1] est basée sur la neutralisation des ions $\mathrm{H}^{+}$par une solution sodique en présence de phénolphtaléine.

\section{1) Principe}

Le lait reconstitué à 5 p. $100(\mathrm{~m} / \mathrm{v})$ est dialysé contre une solution isotonique de chlorure de sodium qui favorise l'ionisation du lait.

Les ions $\mathrm{H}^{+}$diffusent à travers une membrane de cuprophane et décolorent la solution alcaline de phénolphtaléine. Cette technique est donc une méthode de colorimétrie inverse.

La décoloration est mesurée à la longueur d'onde de $580 \mathrm{~nm}$ dans une cuve tubulaire à flux continu de $15 \mathrm{~mm}$. dosage.

La figure 9 représente le montage, ou manifold, particulier à ce 


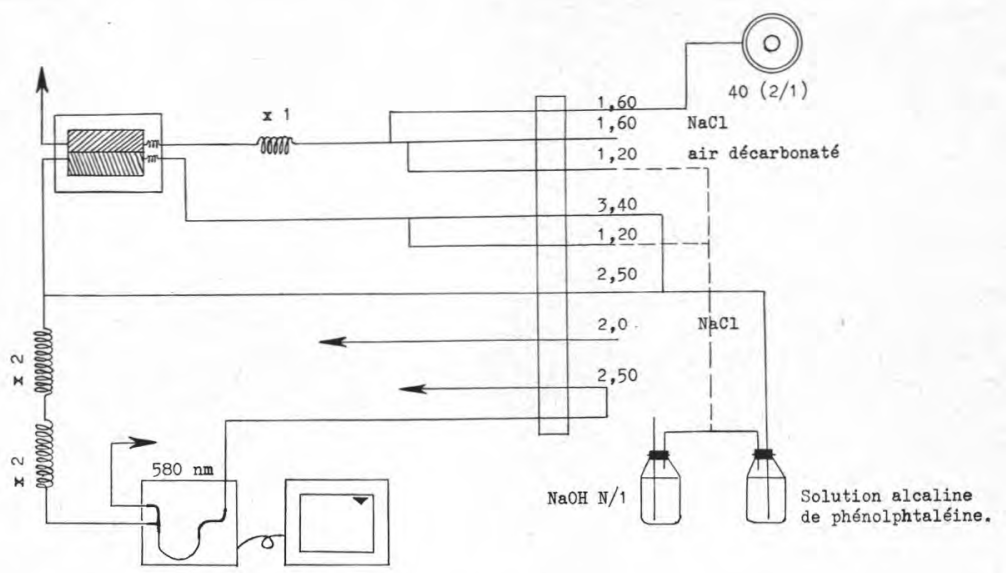

fig. 9

Diagramme des flux pour le dosage de l'acidité titrable

\section{2) Réactifs}

2.1. Solution de chlorure de sodium :

$\mathrm{NaCl}$

Eau distillée q.s.p.

2.2. Solution molaire de carbonate de sodium :

$\mathrm{Na}_{2} \mathrm{CO}_{3}$ anhydre

Eau distillée q.s.p. trique.

Vérifier la molarité de cette solution par un dosage acidimé-

Théoriquement $20 \mathrm{ml} \mathrm{HCl} \mathrm{N}$, en présence d'hélianthine à 1 p. 100 , virent du rouge au jaune avec $10 \mathrm{ml}$ de carbonate de sodium 2,0 N.

2.3. Solution molaire de bicarbonate de sodium :

$\mathrm{NaHCO}_{3}$

Eau distillée q.s.p.

Vérifier la molarité de cette solution.

Théoriquement $20 \mathrm{ml} \mathrm{HCl} \mathrm{N}$, en présence d'hélianthine à 1 p. 100, virent du rouge au jaune avec $20 \mathrm{ml}$ de la solution de bicarbonate $\mathrm{N}$.

\subsection{Mélange tampon:}

Solution molaire de carbonate de sodium (2.2)

Solution molaire de bicarbonate sodium (2.3)

1 vol.

2 vol.

Utiliser ce mélange tampon pour la préparation du réactif coloré. 
2.5 Solution alcaline de phénolphtaléine :

Mélange tampon molaire (2.4) 0,7-0,9 ml

Phénolphtaléine à 1 p. 100 en solution alcoolique $2,0-2,5 \mathrm{ml}$

Eau distillée q.s.p.

$1000 \mathrm{ml}$

2.6 Solution d'hydroxyde de sodium environ normale :

$\mathrm{NaOH}$

$40 \mathrm{~g}$

Eau distillée q.s.p.

\section{3) Etalonnage}

Le lait est un liquide biologique dynamique complexe. L'efficacité de la dialyse, phénomène physique, dépend essentiellement de la quantité et de la nature des protéines. L'étalonnage décrit ici ne peut s'appliquer qu'aux laits secs reconstitués ou aux laits frais dilués.

L'acidité titrable des laits exprimée conventionnellement en acide lactique, est comprise généralement entre 1,3 et 1,8 p. 100. Des laits secs ont été sélectionnés en fonction de leur acidité afin d'obtenir une gamme. Plusieurs déterminations ont été effectuées sur chacun d'eux.

Ces laits sont dilués et traités dans les mêmes conditions que les échantillons à tester.

L'acidité des laits et le réactif n'étant pas stables, il est indispensable de procéder chaque jour à un étalonnage.

La courbe d'étalonnage (fig. 10) est tracée en portant en abscisse, les teneurs en grammes pour $100 \mathrm{~g}$ d'échantillon et en ordonnée, les hauteurs des pics exprimées en p. 100 de transmission.

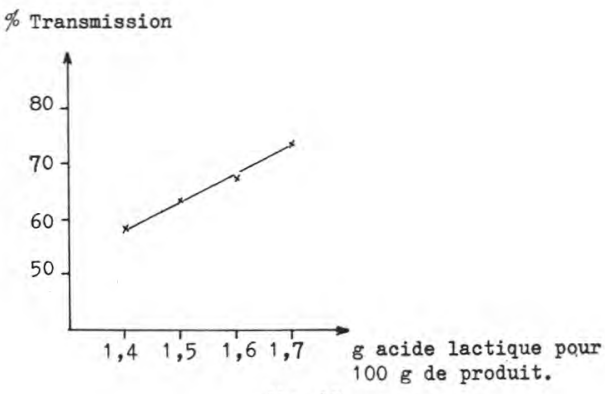

fig. 10

Courbe d'étalonnage du dosage de l'acidité titrable

A partir de cette courbe, on détermine la teneur en acidité lactique des échantillons à tester. 


\section{4) Mise au point de la technique}

\subsection{Choix du réactif}

La composition du réactif coloré doit être telle que la ligne de base correspondant aux réactifs seuls s'établisse à $20 \pm 5$ p. 100 de transmission, le 100 p. 100 de transmission étant réglé sur l'eau.

La figure 11 montre deux exemples des variations obtenues en changeant la composition du réactif de la manière suivante :

Mélange tampon

Solution de phénolphtaléine

de 0,7 à $2,3 \mathrm{ml}$

Eau distillée q.s.p.

de 0,8 à $3,0 \mathrm{ml}$

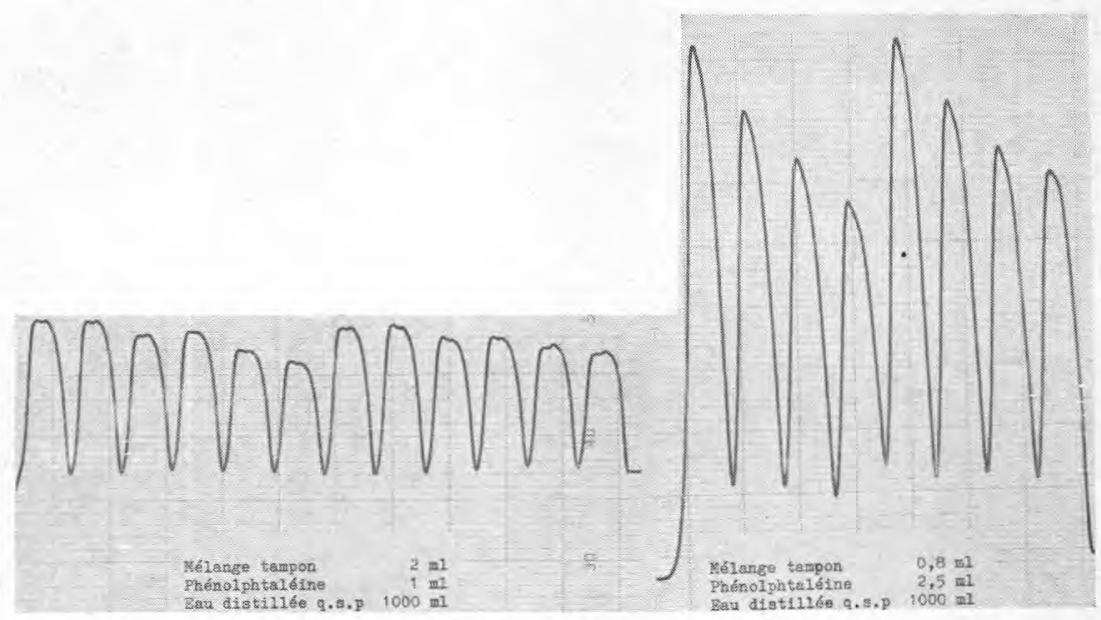

fig. 11

Influence de la concentration du réactif en tampon carbonaté, bicarbonaté et en phénolphtaléine sur la sensibilité de la réaction

\subsection{Cadence analytique}

Afin de déterminer les conditions optimales dans lesquelles peuvent se succéder les prises d'essai, les mêmes échantillons sont analysés dans des conditions identiques, seul le nombre de déterminations par heure varie.

Des essais sont pratiqués à une cadence de $50(2 / 1), 40(2 / 1)$ et 30 (2/1) échantillons/h.

L'examen de la figure 12 nous indique que même à la cadence de 50 échantillons, les pics sont très nettement distincts les uns des autres. La cadence de 40 échantillons/h, donne des résultats précis et répétables. 


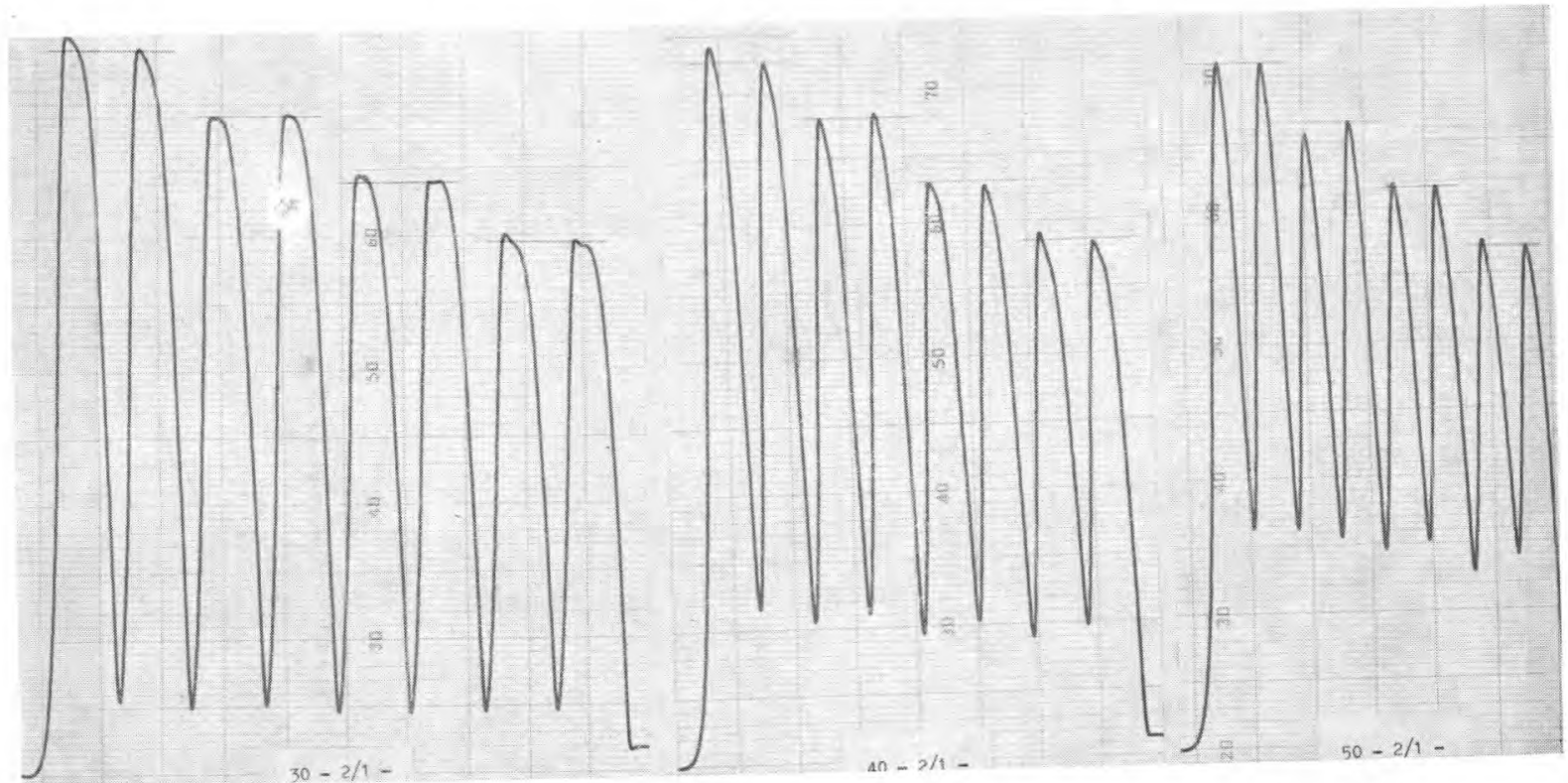

fig. 12

Courbes d'enregistrement des résultats de l'analyse de 4 laits examinés

aux cadences de 30 - 40 - 50 échantillons par heure 


\subsection{Fiabilité de l'appareil}

Un même échantillon présenté à 7 reprises successives, dans les conditions opératoires définies ci-dessus, donne des résultats dont la constance est satisfaisante.

On peut donc conclure que cette méthode de dosage est répétable.

Cependant, nous avons constaté parfois que le premier échantillon d'une série présentait un pic légèrement amplifié. Il suffit de le présenter de nouveau pour avoir sa valeur réelle.

\section{5) Comparaison des méthodes}

Sur 100 échantillons analysés par les deux méthodes, les écarts observés sont les suivants :

\begin{tabular}{c|c}
\hline Nombre d'échantillons & Ecart en p. 100 d'acide lactique \\
\cline { 2 - 2 } 18 & 0 \\
13 & 0,01 \\
13 & 0,02 \\
8 & 0,03 \\
11 & 0,04 \\
13 & 0,05 \\
2 & 0,06 \\
5 & 0,07 \\
3 & 0,08 \\
4 & 0,09 \\
6 & 0,10 \\
1 & 0,11 \\
1 & 0,14 \\
1 & 0,19 \\
1 & 0,21 \\
\hline
\end{tabular}

La corrélation entre les deux méthodes est satisfaisante.

Pour 76 p. 100 des échantillons analysés, l'écart est inférieur ou égal à 0,05 p. 100 d'acide lactique. Pour 96 p. 100 des échantillons, cet écart est inférieur ou égal à 0,1 p. 100 d'acide lactique (fig. 13).

\section{6) Modalités retenues}

6.1 Réactif :

Mélange tampon

Solution de phénolphtaléine

Eau distillée q.s.p.

de 0,7 à $0,9 \mathrm{ml}$ de 2,0 à $2,5 \mathrm{ml}$ $1000 \mathrm{ml}$

\subsection{Cadence : $40(2 / 1)$.}

Observations : L'air qui sert de gaz de segmentation doit être décarbonaté ; le faire barboter dans une solution d'hydroxyde de sodium normale. 


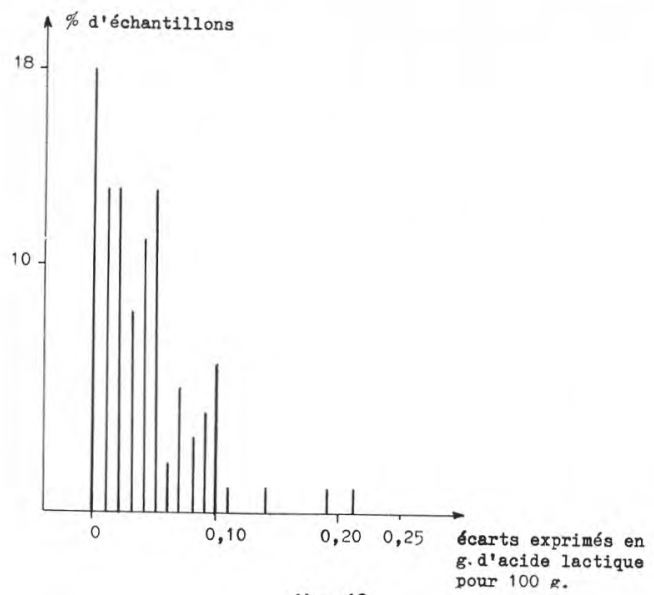

fig. 13

Répartition des écarts entre les résultats des deux méthodes analytiques

Nous avons remarqué que les laits riches en sodium, potassium, calcium et sels ammoniacaux, donnent une réponse par la méthode automatique, différente de celle obtenue par la méthode de référence [1]. La teneur anormalement élevée de ces cations entraîne des interférences dans la réaction, ce qui permet de suspecter la présence de neutralisant. (On sait que tout échantillon suspect est soumis à des épreuves complémentaires de détermination directe des cations).

L'acidité des lactosérums dont la composition diffère des laits secs, peut être déterminée selon le même principe à condition de procéder à un étalonnage approprié.

\section{III. - DETERMINATION DE LA TENEUR EN AZOTE AMMONIACAL DES LAITS ET LACTOSERUMS SECS ECREMES}

Nous nous sommes référés pour ce dosage, à la méthodologie proposée par la Société Technicon, pour le dosage de l'ammoniémie $[5,6]$ basée sur la réaction de Berthelot. Cette technique s'adapte plus facilement à l'analyse automatique que la méthode de référence [1] qui nécessite le déplacement de l'ammoniac par entraînement à la vapeur d'éthanol. 


\section{1) Principe}

Les molécules d'ammoniac du lait reconstitué diffusent à travers la membrane de cuprophane et donnent de l'indophénol en présence d'hypochlorite et de phénate de sodium en milieu alcalin, à une température voisine de $60^{\circ} \mathrm{C}$.

La coloration bleue de l'indophénol est mesurée à la longueur d'onde de $630 \mathrm{~nm}$ dans une cuve tubulaire à flux continu de $15 \mathrm{~mm}$.

La figure 14 montre le montage particulier à ce dosage.

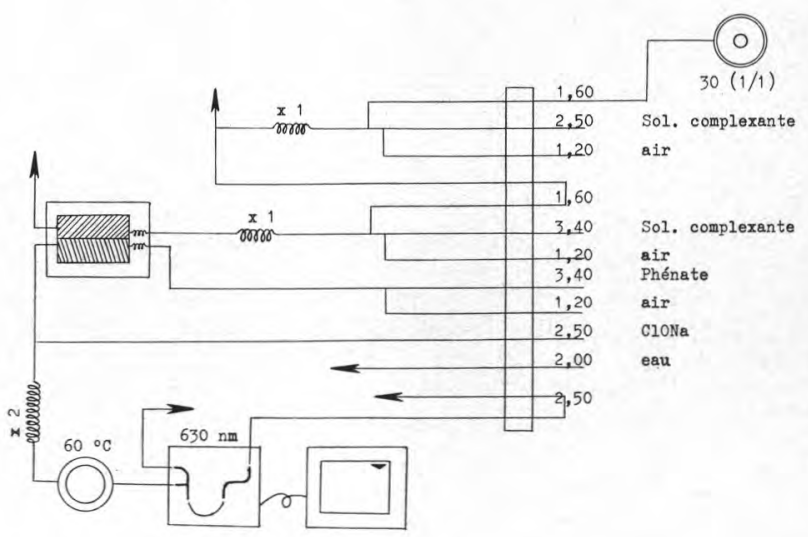

fig. 14

Diagramme des flux pour le dosage de l'azote ammoniacal

\section{2) Réactifs}

\subsection{Solution complexante :}

$\mathrm{NaOH}$ en pastilles

Tartrate de $\mathrm{Na}$ et de $\mathrm{K}$

Citrate trisodique

Eau distillée q.s.p.

2.2. Solution de phénate de sodium:

Phénol cristallisé

$140 \mathrm{ml}$

$\mathrm{NaOH}$ en pastilles

Eau distillée q.s.p.

2.3. Solution d'hypochlorite de sodium:

$\mathrm{ClONa}$ à $10^{\circ}$ chlorométriques français Eau distillée q.s.p. 
2.4. Solution de référence de sulfate d'ammonium :

1) Solution mère $A$.

Faire dissoudre $38,8647 \mathrm{~g}$ de $\left(\mathrm{NH}_{4}\right)_{2} \mathrm{SO}_{4}$ dans de l'eau distillée. Amener à $1000 \mathrm{ml}$ avec de l'eau distillée.

$1 \mathrm{ml}$ de cette solution contient l'équivalent de $10 \mathrm{mg}$ d'ammoniac.

2) Solutions diluées B, C, D... H.

A partir de la solution mère, préparer des solutions diluées en prélevant $\mathrm{X} \mathrm{ml}$ de la solution $\mathrm{A}$ et en complétant à $100 \mathrm{ml}$ à l'aide d'eau distillée.

Préparer, par exemple, les solutions suivantes :

Solution B : $3 \mathrm{ml} / 100 \mathrm{ml}$ soit $300 \mathrm{ng} \mathrm{NH}{ }_{3}$ par $\mathrm{ml}$.

Solution C : $6 \mathrm{ml} / 100 \mathrm{ml}$ soit $600 \mathrm{ng} \mathrm{NH}$ par ml.

Solution $\dot{\mathrm{H}}$ : $21 \dot{\mathrm{ml}} / 100 \mathrm{ml}$ soit $2100 \mathrm{ng} \mathrm{NH}_{3}$ par ml.

\section{3) Etalonnage}

A un lait sec, reconstitué à 5 p. $100(\mathrm{~m} / \mathrm{v})$, exempt d'ammoniac ou de teneur aussi faible que possible, ajouter des quantités croissantes de sulfate d'ammonium.

Préparation de la courbe d'étalonnage

- Echantillon témoin

$1 \mathrm{~g}$ de lait sec (défini précédemment) est dilué avec $20 \mathrm{ml}$ d'eau distillée.

- Echantillons d'étalonnage

Introduire dans une série de 7 tubes, $1 \mathrm{~g}$ du lait sec précédent.

Ajouter $19 \mathrm{ml}$ d'eau distillée et agiter.

Ajouter respectivement dans chaque tube, $1 \mathrm{ml}$ de chacune des solutions B à $\mathrm{H}$.

Ces 9 échantillons servent à l'établissement de la courbe d'étalonnage (fig. 15). Ils sont traités dans les mêmes conditions que les échantillons à tester.

\section{4) Mise au point de la technique}

\subsection{Choix du réactif}

Dans cette technique, la sensibilité de la réaction dépend essentiellement de la concentration en phénate de sodium. 


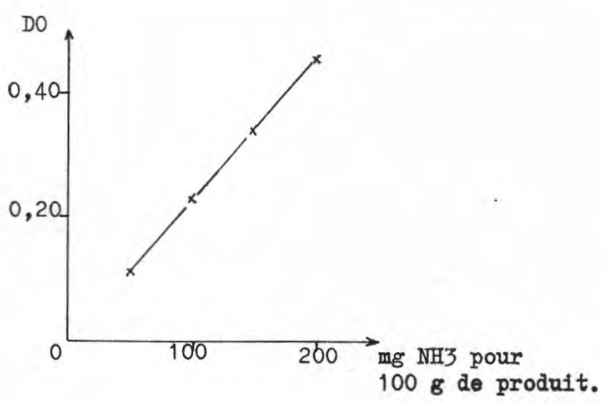

fig. 15

Profil de la courbe d'étalonnage du dosage de l'azote ammoniacal

Nous avons étudié à une température réactionnelle de $58^{\circ} \mathrm{C}$ (voir chapitre 4.2), au rythme analytique de 30 échantillons/h (voir chapitre 4.3) les différences d'amplitude en fonction de la teneur en phénate de sodium de la solution.

Les réactifs ont été préparés de la façon suivante, après fusion du phénol cristallisé :

\begin{tabular}{l|r|r|r|r|r}
\hline Réf. figure 16 & a & b & c & d & e \\
\cline { 1 - 2 } & & & & & \\
Phénol, en ml & 80 & 100 & 116 & 140 & 160 \\
NaOH, en g & 60 & 65 & 67,5 & 80 & 80 \\
Eau distillée q.s.p. en ml & 1000 & 1000 & 1000 & 1000 & 1000 \\
\hline
\end{tabular}

La loi de Beer-Lambert n'est plus respectée comme l'indique la figure 16 lorsque la concentration en phénate de sodium est trop élevée (e).

Parmi les cinq solutions étudiées, la solution (d) donne l'amplitude maximale tout en respectant la loi de Beer-Lambert.

\subsection{Température}

Un autre essai porte sur la recherche de la température optimale de la réaction de Berthelot.

Les échantillons sont traités selon le même mode opératoire, seule la température de réaction (température du bain d'huile), varie de $42^{\circ} \mathrm{C}$ à $70^{\circ} \mathrm{C}$ (fig. 17). 


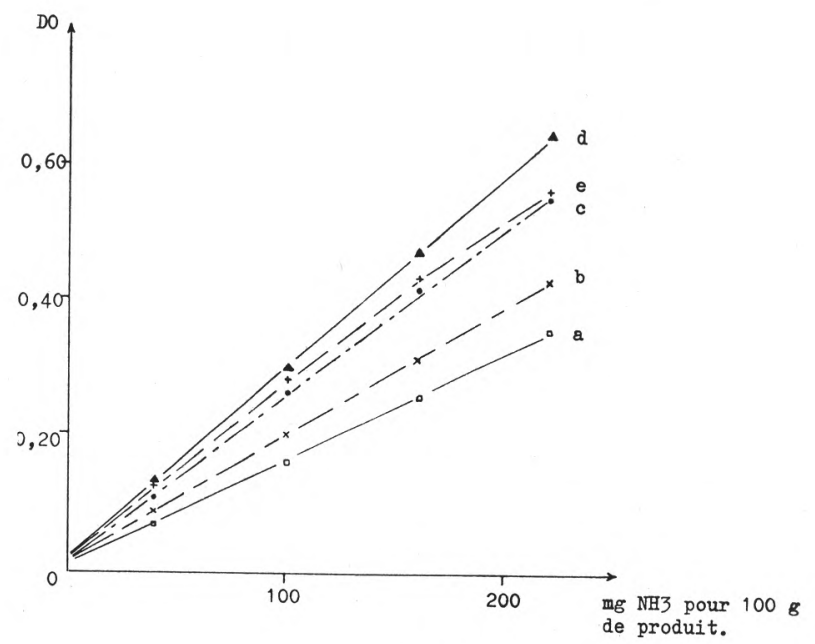

fig. 16

Influence de la concentration en phénate de sodium sur la sensibilité de la réaction

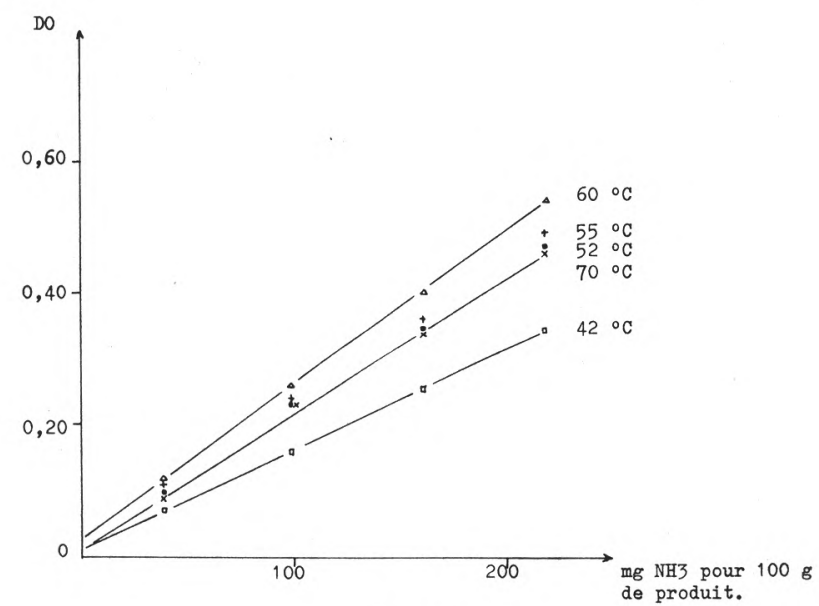

fig. 17

Influence de la température sur le développement de la coloration 
Aux températures extrêmes de l'essai, on enregistre une diminution de sensibilité. Le développement maximal de la coloration due à la réaction de Berthelot est observé à $60^{\circ} \mathrm{C}$.

\subsection{Cadence analytique}

Les échantillons de laits reconstitués sont analysés à une cadence de : $20(1 / 1), 30(1 / 1), 40(2 / 1)$.

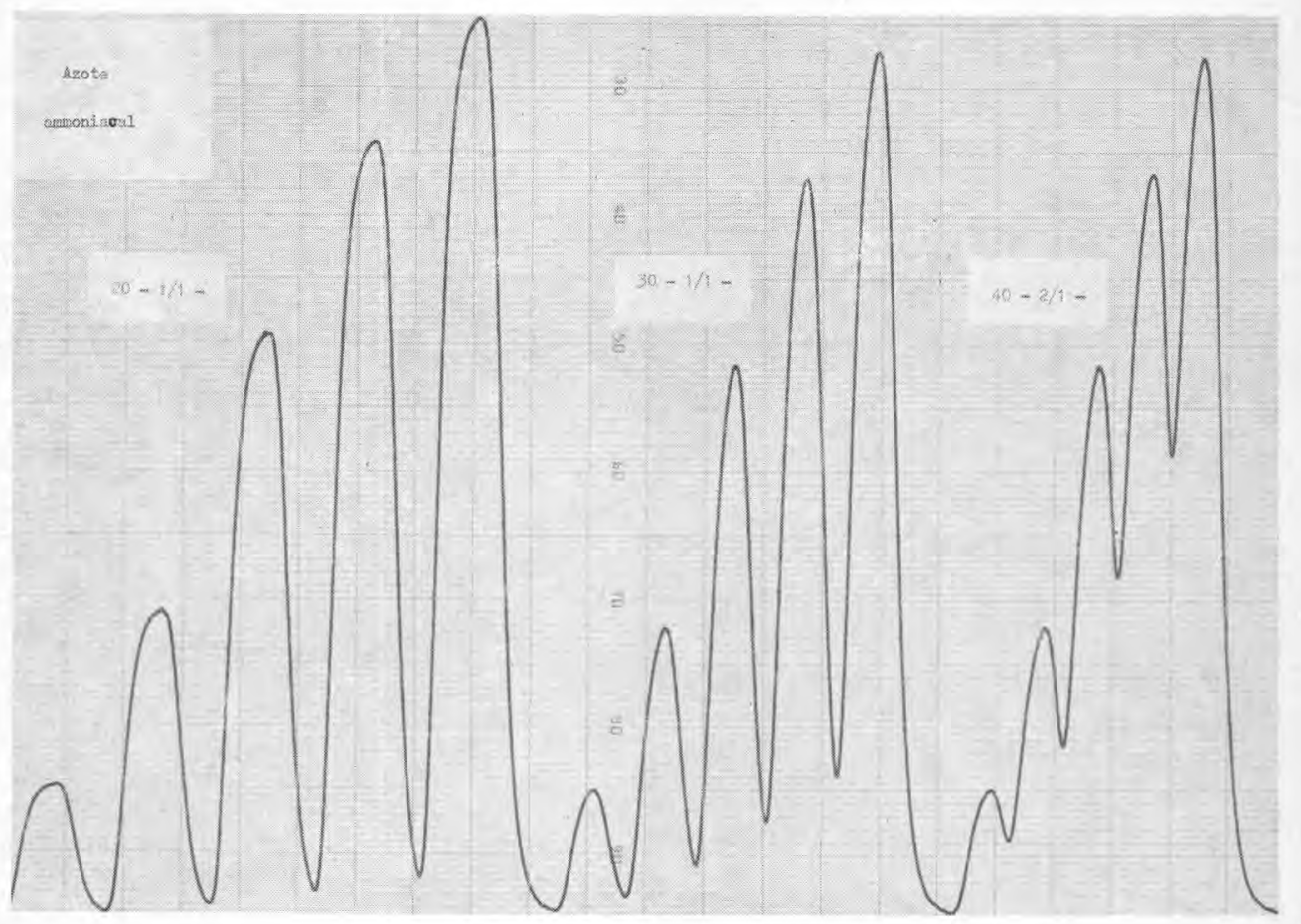

fig. 18

Graphique d'enregistrement d'une gamme étalon d'azote ammoniacal au rythme de 20 - 30 - 40 échantillons/h

L'amplitude des pics diminue sensiblement quand la cadence des déterminations augmente. Une cadence de 30 déterminations $/ \mathrm{h}$ donne une bonne séparation entre les différents pics. A 40 échantillons/h, le rinçage risque d'être insuffisant : un échantillon riche en ammoniac peut contaminer le suivant et même masquer le pic d'un lait ayant une teneur faible en azote ammoniacal (fig. 18). 


\subsection{Fiabilité de l'appareil}

Un même échantillon présenté à 7 reprises successives, dans les conditions opératoires définies ci-dessus, donne des résultats dont la constance est très satisfaisante.

En effet, les faibles variations d'amplitude enregistrées n'entraînent pas de différences significatives entre les teneurs en ammoniac.

\section{5) Comparaison des méthodes}

Sur les 80 échantillons, essentiellement des lactosérums secs écrémés, analysés par la méthode de référence [1] et par la méthode automatisée sur l'appareil Technicon, nous avons constaté :

\begin{tabular}{c|c}
\hline Nombre d'échantillons & Ecart en $\mathrm{mg} \mathrm{NH}_{3}$ pour $100 \mathrm{~g}$ \\
& \\
18 & 0 \\
5 & 1 \\
9 & 2 \\
4 & 3 \\
2 & 4 \\
0 & 5 \\
1 & 6 \\
4 & 7 \\
2 & 8 \\
6 & 9 \\
1 & 10 \\
1 & 12 \\
1 & 13 \\
1 & 14 \\
1 & 18 \\
& 24 \\
\hline
\end{tabular}

La moyenne des écarts relevés est égale à 3,56 mg d'azote ammoniacal pour $100 \mathrm{~g}$ de produit.

L'examen de l'histogramme (fig. 19) indique l'écart entre les deux méthodes pratiquées sur les 80 échantillons :

$-77 \mathrm{p} .100 \leqslant$ à $5 \mathrm{mg} \quad$ pour $100 \mathrm{~g}$,

- $16 \mathrm{p} .100$ entre $5 \mathrm{mg}$ et $10 \mathrm{mg}$ pour $100 \mathrm{~g}$,

- 4 p. 100 entre $10 \mathrm{mg}$ et $15 \mathrm{mg}$ pour $100 \mathrm{~g}$,

- 3 p. 100 entre $15 \mathrm{mg}$ et $25 \mathrm{mg}$ pour $100 \mathrm{~g}$.

Il faut noter que les différences supérieures à $10 \mathrm{mg}$ ont été obtenues sur des échantillons de lactosérum ayant des teneurs en azote ammoniacal supérieures à $100 \mathrm{mg}$ pour $100 \mathrm{~g}$ de produit.

Toutefois, en pratique courante, lorsque les résultats risquent de déclasser le produit analysé, le dosage est toujours renouvelé. 


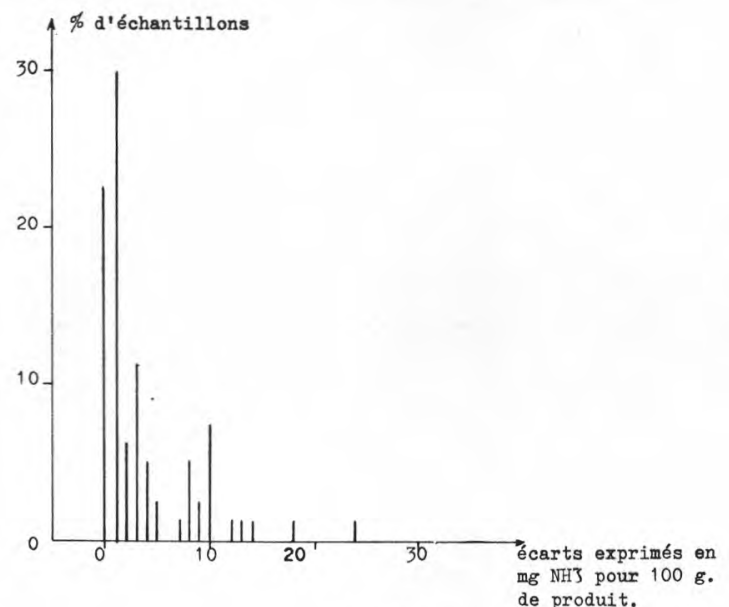

fig. 19

Répartition des écarts entre les résultats des deux méthodes analytiques

\section{6) Modalités retenues}

6.1. Réactif :

Phénol

$\mathrm{NaOH}$

Eau distillée q.s.p.

\subsection{Température}

La température doit être constante pendant tout l'essai ; elle peut être choisie entre $55^{\circ} \mathrm{C}-60^{\circ} \mathrm{C}$.

6.3. Cadence : $30(1 / 1)$.

Observations : La manipulation de flacons d'ammoniaque dans la salle de travail est à proscrire. Il faut opérer dans une ambiance dépourvue d'émanations de produits azotés et protéger au maximum les prises d'essai d'une contamination éventuelle.

La détermination du taux d'azote ammoniacal des laits et lactosérums secs se pratique dans les mêmes conditions. La teneur en protéines des échantillons n'interfère plus au moment de la dialyse, car la solution est 40 fois plus diluée que pour la détermination de l'acidité titrable et de l'acide lactique/lactates.

En conséquence, à partir d'un seul étalonnage, la détermination du taux d'azote ammoniacal s'effectue sur des laits et des lactosérums secs après reconstitution à 5 p. 100 (m/v). 


\section{CONSIDERATIONS ECONOMIQUES}

L'acquisition d'un autoanalyseur constitue un investissement important. Le prix d'achat de l'appareil avec les accessoires utilisés était de l'ordre de $60000 \mathrm{~F}$ en 1969.

L'entretien de l'appareillage ne nous a pas occasionné de frais particuliers au cours des années écoulées.

Le coût des réactifs est minime comme le montre le tableau cidessous établi pour l'analyse de 1000 échantillons :

- Détermination de l'acide lactique/lactates :

$\bullet \simeq 7,5 \mathrm{ml}$ perchlorure de fer,

- $\simeq 100 \mathrm{~g}$ de chlorure de sodium.

- Détermination de l'acidité titrable :

- quelques g de carbonate de sodium, de bicarbonate de sodium et de phénolphtaléine,

$-\simeq 55 \mathrm{~g}$ de chlorure de sodium.

- Détermination de l'azote ammoniacal :

- $\simeq 750 \mathrm{ml}$ phénol,

$-\simeq 200 \mathrm{~g}$ tartrate de sodium et potassium,

- $\simeq 600 \mathrm{~g}$ hydroxyde de sodium,

$\because \simeq 50 \mathrm{~g}$ citrate trisodique,

$-\simeq 1500 \mathrm{ml}$ hypochlorite de sodium.

Le prix d'achat des produits nécessaires à ces trois analyses est estimé à $14,70 \mathrm{~F}$ pour 1000 échantillons. En tenant compte des fournitures diverses (godets, tubes de pompe, papier, encre, etc.), ce montant s'élève à moins de $500 \mathrm{~F}$.

Par ailleurs, il apparaît inutile d'insister sur l'économie énorme réalisée sur la verrerie de laboratoire et son entretien.

\section{CONCLUSION}

Notre étude a montré que l'autoanalyse convient parfaitement à un laboratoire chargé du contrôle, en grande série, de produits laitiers. L'autoanalyse est vraisemblablement appelée à se généraliser.

La transposition des méthodes traditionnelles nécessite une adaptation particulière tenant compte des impératifs inhérents à l'appareil. Jusqu'à présent, celui-ci ne peut traiter que des échantillons liquides ; les produits secs doivent être dilués. La reconstitution est la seule opération manuelle à effectuer.

Les avantages de l'automation du dosage de l'acide lactique/lactates, de l'acidité titrable, de l'azote ammoniacal sont techniquement et financièrement démontrés. Elle permet l'obtention de résultats aussi précis et répétables que les méthodes manuelles. 
Ces analyses se trouvant effectuées par l'autoanalyseur, le personnel peut se consacrer à d'autres tâches. D'autre part, cet appareil peut apporter des informations complémentaires pour la définition de la qualité des produits laitiers.

\section{R és u m é}

Automation des déterminations dans les laits et les lactosérums de l'acide lactique/lactates par coloration au perchlorure de fer, de l'acidité titrable par décoloration d'une solution tamponnée de phénolphtaléine et de l'azote ammoniacal par formation d'indophénol.

Mise au point de ces différentes techniques sur l'autoanalyseur I Technicon.

\section{S u m m a ry}

Automation of determination in milk and whey of lactic acid/ lactates using ferric chloride coloration, of titrable acidity by decoloration of buffered phenolphtalein solution and of ammoniacal azote through indophenol formation.

Adaptation of the various techniques to the Technicon autoanalyzer I.

\section{Remerciements}

Nous remercions Mme Serres, Directrice du Laboratoire, pour les suggestions et critiques faites pour la réalisation de ce travail.

Reçu pour publication le 26 mars 1973.

\section{Références bibliographiques}

[1] Serres (L.), Amariglio (S.) et Petransxiene (D.). - Contrôle de la qualité des produits laitiers (tome I, Analyses physique et chimique), édité par Informations Techniques des Services Vétérinaires.

[2] Dosage du gaz carbonique. Méthodologie Technicon, novembre 1966.

[3] Serres (L.), Amariglio (S.), Benard (L.) et Robinet (D.) (1960). - Contribution à l'étude des méthodes de recherche des neutralisants dans les poudres de lait écrémé, Le Lait, $\mathrm{n}^{\circ}$ 393-394 et n 395-396.

[4] BRET (1964). - Utilisation de l'appareillage Technicon dans l'industrie fromagère du Roquefort. Symposium Technicon 1960.

[5] Dosage de l'ammoniac. Méthodologie Technicon.

[6] Agneray (J.) (1961). - Dosage automatique de l'azote ammoniacal dans les minéralisats. Annales de Biologie Clinique, $\mathrm{n}^{\circ}$ 1-2. 